\title{
Sezgisel önermeler lojiği için semantikler
}

\author{
Süleyman POLAT, Mehmet TERZİLER* \\ Yaşar Üniversitesi Fen-Edebiyat Fakültesi Matematik Bölümü, İzmir. \\ Geliş Tarihi (Recived Date): 03.04.2018 \\ Kabul Tarihi (Accepted Date): 12.06.2018
}

\section{Özet}

Bu çalışmada Sezgisel Önermeler Lojiği (IPL) için üç semantik sunuyoruz; yani Kripke semantiği, Heyting semantiği ve topolojik semantik. IPL'nin bu semantiklere göre sağlam ve tam olduğunu gösteriyoruz.

Anahtar Kelimeler: Sezgisel lojik, Kripke semantiği, modal lojik, Heyting cebirleri, topolojik semantik.

\section{Semantics for intuitionistic propositional logic}

\begin{abstract}
In this study we present three semantics for Intuitionistic Propositional Logic (IPL); namely, Kripke semantics, Heyting semantics, and topological semantics. We show that IPL is sound and complete with respect to these semantics.
\end{abstract}

Keywords: Intuitionistic logic, Kripke semantics, modal logic, Heyting algebras, topologic semantics.

\section{Giriş}

Lojiğin klasik anlayışı doğruluk kavramına dayanır; bir önermenin doğruluğu mutlaktır ve her hangi bir akıl yürütme ya da eylemden bağımsızdır. Bir önerme ya doğrudur yada yanlıştır; yanlış doğru olmayan ile aynı anlama sahiptir ve bu aşağıdaki üçüncünün olmazlığı yasası (tertium non datur ya da excluded middle) olarak ifade edilir:

“p önermesinin anlamı ne olursa olsun, $p \vee \neg p$ önermesi her zaman doğrudur.”

Süleyman POLAT, polat3635@ @otmail.com, https://orcid.org/0000-0002-5418-1412

* Mehmet TERZILER, mehmet.terziler@yasar.edu.tr, https://orcid.org/0000-0002-0837-474X 
Ancak $p \vee \neg p$ önermesi sınırlı bilgi içerir. Bunu açıklayan iki iyi bilinen örnek verilebilir. İlki,

“ $\pi$ sayısının ondalık gösteriminde bir yerde yedi tane 7 art arda bulunur.”

Bu önermenin doğruluk değerinin belirlenmesi kesin değildir. İkincisi,

$$
\text { “ } x^{y} \text { rasyonel olacak şekilde } x \text { ve y irrasyonel saylları vardır." }
$$

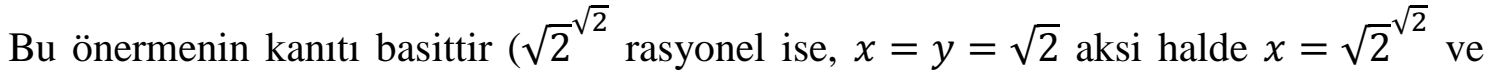
$y=\sqrt{2}$ almak yeterlidir), ancak iki durumdan hangisinin doğru olduğu bilinmemektedir.

$\mathrm{Bu}$ örnekler klasik önermeler lojiğinin kusurlarından bazılarını açığa çıkarır ve neden sezgisel (intuitionistic) ya da inşacı (constructive) lojiğe ilgi duyulması gerektiğinin ipuçlarını verir. Sezgisel önermeler lojiği (IPC), klasik önermeler lojiğinden (CP) üçüncünün olmazlığı yasası ve olmayana ergi kuralı (reductio ad absurdum) çıkarılarak tanımlanabilir; o zaman CP nin zayıflatılmış bir halidir ve bunun sonucu olarak CP ye göre daha geniş semantik yorum aralığına sahiptir. Motive edici semantiğe sezgisel önermeler lojiğinin Brouwer-Heyting-Kolmogorov (BHK) yorumu denir. Sezgisel lojiğin ortaya atılışını ve esaslarını ayrıntılı incelemek için $[1,9,10]$ kaynaklarına bakılabilir.

$\mathrm{Bu}$ çalışmada IPC için üç model tartışılıyor: Kripke yapıları, Heyting cebirleri ve topolojik modeller. Modellerin özellikleri ile birlikte Kripke yapıları ve Heyting cebirleri arasındaki ilişki inceleniyor. Konu ile ilgili olarak [2, 5, 8] kaynaklarına bak1labilir.

\section{2. Ön bilgiler}

Klasik önermeler lojiğinin bir formülünün standart semantiği, formüldeki önerme değişkenlerine $\mathbf{2}=\{0,1\}$ Boole cebrinde ya da iki elemanlı diğer her hangi bir Boole cebrinde bir doğruluk değeri atanarak tanımlanır. $\mathbb{P}=\{p, q, \quad r, \ldots\}$ sonsuz sayılabilir çoklukta önerme değişkenlerinin kümesini göstermek üzere böyle bir atama $V: \mathbb{P} \rightarrow \mathbf{2}$ fonksiyonu ile yapılır ve bu fonksiyona bir değerlendirme (valuation) fonksiyonu denir. $\quad \mathbb{F}_{C}$ önermeler lojiğinin formüller kümesini göstermek üzere $V$ fonksiyonu $\mathbb{F}_{C}$ kümesine aşağıdaki gibi genişletilebilir: Her $\varphi, \psi \in \mathbb{F}_{C}$ için

$$
\begin{aligned}
& V(\neg \varphi)=\neg V(\varphi) \\
& V(\varphi \wedge \psi)=V(\varphi) \wedge V(\psi) \\
& V(\varphi \vee \psi)=V(\varphi) \vee V(\psi) \\
& V(\varphi \rightarrow \psi)=V(\varphi) \rightarrow V(\psi) .
\end{aligned}
$$

Burada $\wedge, \vee, \rightarrow, \mathbb{P}$ üzerinde önerme bağlaçları ve $\neg$ değilleme işlemidir. Gerektiğinde, $\neg \varphi, \varphi \rightarrow \perp$ olarak alınacaktır ve $\perp$ yanlışı, T ise doğruyu gösterecektir. 
Her $V: \mathbb{P} \rightarrow \mathbf{2}$ için $V(\varphi)=\mathrm{T}$ (ya da 1 ) ise $\varphi$ formülüne geçerli (valid) formül denir. Klasik önermeler lojiğinin Tamlık Teoremi (Completeness Theorem)'ne göre $\varphi$ formülü CP'de kantlanabilirdir ancak ve ancak $\varphi$ formülü geçerlidir. 2 Boole cebiri yerine keyfi bir $\mathbb{B}$ Boole cebiri alınabilir ve değer atama kavramı bu cebire genişletilebilir. Her $V: \mathbb{P} \rightarrow \mathbb{B}$ fonksiyonu için $V(\varphi)=\mathrm{T}$ ise $\varphi$ formülü $\mathbb{B}$-geçerlidir denir. Şimdi $\mathrm{CP}$ lojiği için iyi bilinen tamlık teoreminin aşağıdaki versiyonu ifade edilebilir:

$\varphi$ formülü $\mathrm{CP}$ lojiğinde kanıtlanabilirdir ancak ve ancak her $\mathbb{B}$ Boole cebiri için $\mathbb{B}$ geçerlidir.

Aynı şekilde IPC için bir semantik tanımlanabilir, ancak bu durumda doğruluk değerleri Boole cebiri yerine bir $\mathbb{H}$ Heyting cebirine ait olacaktır. Bir $\mathbb{H}$-değerlendirme bir $V: \mathbb{P} \rightarrow \mathbb{H}$ fonksiyonudur ve formüllerin kümesine genişletilebilir. Her $V: \mathbb{P} \rightarrow \mathbb{H}$ değerlendirmesi için $V(\varphi)=\mathrm{T}$ ise $\varphi$ formülü $\mathbb{H}$-geçerlidir denir.

Tanım 2.1 (Heyting gerektirme) $\Gamma$ sonlu bir formüller kümesi ve $\varphi$ bir formül olsun. Her $V \mathbb{H}$-değerlendirmesi için $V(\Lambda \Gamma) \leq V(\varphi)$ ise $\Gamma, \varphi$ formülünü Heyting gerektirir ya da $\Gamma, \varphi$ nın bir $\mathbb{H}$-sonucudur denir ve $\Gamma \vDash \mathbb{H} \varphi$ ile gösterilir.

Notasyon 2.2 $\mathrm{Bu}$ tanımda $\Gamma=\left\{\varphi_{1}, \varphi_{2}, \ldots, \varphi_{n}\right\}$ ise $\wedge \Gamma, \varphi_{1} \wedge \varphi_{2} \wedge \ldots \wedge \varphi_{n}$ ifadesinin kısa yazılışıdır. Ayrıca sezgisel önermeler lojiği IPC ya da Int olarak ve önermeler lojiği CP olarak kısaltılacaktır.

Sezgisel önermeler lojiği, $\varphi \vee \neg \varphi$ ya da $\neg \neg \varphi \rightarrow \varphi$ çifte değilleme yasası olmadan kısaca klasik önermeler lojiği olarak tanımlanabilir. Aksiyomları ve kuralları aşağıdaki gibidir:

Aksiyomlar $\varphi, \psi \in \mathbb{F}_{C}$ için

- $\varphi \rightarrow(\psi \rightarrow \varphi)$

- $(\varphi \rightarrow(\psi \rightarrow \chi)) \rightarrow((\varphi \rightarrow \psi) \rightarrow(\varphi \rightarrow \chi))$

- $\varphi \rightarrow(\psi \rightarrow \varphi \wedge \psi)$

\section{Kurallar}

$$
\begin{aligned}
& \frac{\varphi \psi}{\varphi \wedge \psi}(\wedge I) \quad \frac{\varphi \wedge \psi}{\varphi}(\wedge E 1) \quad \frac{\varphi \wedge \psi}{\psi}(\wedge E 2) \\
& \frac{-h}{\varphi} \\
& \vdots \\
& \frac{\psi}{\varphi \rightarrow \psi}(\rightarrow I, h) \quad \frac{\varphi \rightarrow \psi \quad \varphi}{\psi}(\rightarrow E \text { yada MP) } \\
& \frac{\varphi}{\varphi \vee \psi}(\vee I 1) \quad \frac{\psi}{\varphi \vee \psi}(\vee I 2) \quad \frac{\perp}{\varphi}(\perp E)
\end{aligned}
$$




\section{Boole cebirleri ve Heyting cebirleri}

Burada Boole cebiri ve Heyting cebirinin tanımları hatırlatılmakla birlikte her iki cebir için farklı ancak denk tanımların verilebildiği gösteriliyor.

\subsection{Boole cebirleri}

Bir Boole cebiri $\mathbb{B}=\langle B, \wedge, \vee, \quad$, $\quad 0,1\rangle$ genellikle tipli bir yapı olarak tanımlanır öyle ki $B$ üzerinde tanımlanan $\vee$ ve $\wedge$ ikili işlemleri birleşmeli, değişmeli, birbiri üzerine dağılmalı ve eşgüçlü (idempotent) dür; birli işlem ' çifte değillemeyi (her $b \in B$ için $\left(b^{\prime}\right)^{\prime}=b$ ) sağlar ve 0,1 elemanları sırasıyla en küçük ve en büyük elemanlardır. Bir Boole cebiri kafes (lattice) kavramı aracılığıyla da tanımlanabilir.

Tanım 3.1.1 Bir kafes $, \mathbb{L}=\langle L, \quad \vee, \quad \wedge\rangle,(2,2)$ tipli bir yapıdır öyle ki her $a, b, c \in L$ için aşağıdaki aksiyomlar sağlanır:
$(\mathrm{L} 1)(a \vee b) \vee c=a \vee(b \vee c)$
$(\mathrm{L} 1)^{\partial}(a \wedge b) \wedge c=a \wedge(b \wedge c)$
(L2) $a \vee b=b \vee a$
$(\mathrm{L} 2)^{\partial} a \wedge b=b \wedge a$
(L3) $a \vee a=a$
$(\mathrm{L} 3)^{\partial} a \wedge a=a$
$(\mathrm{L} 4) a \vee(a \wedge b)=a$
$(\mathrm{L} 4)^{\partial} a \wedge(a \vee b)=a$

(L3) ve (L3) eşgüçlülüik aksiyomlarının her biri (L4) ve (L4) yutma (absorption) aksiyomlarından türetilebilir ancak bir kafesi Tanım 3.1.1 altında tanımlamak gelenektir. $L$ üzerinde $a \leq b \Leftrightarrow a \vee b=b$ ya da $a \wedge b=a$ gibi bir sıralama bağıntısı tanımlanabilir.

Tanım 3.1.2 $L$ bir kafes olsun. Her $a \in L$ için $a=a \vee 0$ olacak şekilde bir sıfir elemanı $0 \in L$ varsa ve her $a \in L$ için $a=a \wedge 1$ olacak şekilde bir bir (ya da birim) elemanı $1 \in L$ varsa, $L$ kafesine sinurlı kafes denir.

Tanım 3.1.3 Bir dağılmalı kafes aşağıdaki dağılma aksiyomlarından birini sağlayan kafestir. Her $a, b, c \in L$ için

$$
(D) a \wedge(b \vee c)=(a \wedge b) \vee(a \wedge c) \quad(D)^{\partial} a \vee(b \wedge c)=(a \vee b) \wedge(a \vee c)
$$

Tanım 3.1.4 $L$ sinırlı bir kafes ve $a \in L$ olsun. Eğer $a \wedge b=0$ ve $a \vee b=1$ ise $b \in L$ elemanı $a$ nın bir tümleyenidir. $a$ elemanının tek bir tümleyeni varsa o tümleyen $a^{\prime}$ ile gösterilir. Her elemanı tek tümleyene sahip bir kafese tümlenmiş kafes denir.

Nihayet bir Boole cebiri kafes açısından şöyle tanımlanabilir:

Tanım 3.1.5 Bir Boole cebiri sınırlı, dağılmalı ve tümlenmiş bir kafestir.

Tanım 3.1.1 deki her bir aksiyom düale sahiptir ve CP nin iyi bilinen Düalite İlkesi gereği $\vee$ ve $\wedge$ işlemlerinden birinden vazgeçilebilir. Böylece Boole cebirlerinin yeni bir tanımı yapılabilir. Ayrıntı için [3] kaynağına bakılabilir. 
Tanım 3.1.6 Bir Boole cebiri aşağıdaki koşulu sağlayan $(2,1)$ tipli bir $\mathbb{B}=\left\langle B, V,{ }^{\prime}\right\rangle$ yapisidir:

$u \in B$ elemanı vardır öyle ki

1. $B$ üzerinde $p^{\prime} \vee q=u$ olarak tanımlanan $p \leq q$ bağıntısı bir sıralama bağıntısı olsun.

2. $p \vee q=\sup \{p, q\}$

Böyle bir $u \in B$ elemanı varsa, her $p \in B$ için $u=p^{\prime} \vee p$ olduğu için $u$ tektir. Bu tek elemana birim denir ve 1 ile gösterilir.

Teorem 3.1.7 Tanım 3.1.5 ve Tanım 3.1.6 denktir.

Kanıt [3] de verilmiştir.

\subsection{Heyting cebirleri}

Bir Boole cebiri tümleyen işlemi zayıflatılarak genelleştirilebilir. Bunu yerine getirmenin bir yolu sözde tümleyeni (pseudo-complement) tanımlamaktan geçer.

$L$, 0 elemanlı bir kafes ve $a \in L$ olsun. $a^{*}=\max \{b \in L: b \wedge a=0\}$, $a$ nın sözde tümleyeni olsun. Aşağıda verilen Heyting cebiri tanımında $\rightarrow$ gerektirme işlemi $a^{*}=a \rightarrow 0$ sözde tümleyen kavramı ile ilişsilendirilebilir.

Tanım 3.2.1 Bir Heyting kafesi ya da Heyting cebiri

$$
c \leq a \rightarrow b \text { ancak ve ancak } a \wedge c \leq b
$$

şeklinde gerektirme adı verilen bir $\rightarrow$ ikili işleme sahip sınırlı ve dağılmalı bir kafestir.

Bir Heyting cebiri; $\vee, \wedge$ ve $\rightarrow$ işlemlerinin belirli aksiyomları sağladığı 0 ve 1 elemanlı bir $\mathbb{H}=\langle H, \leq\rangle$ kısmi sıralı yapı olarak da tanımlanabilir. Ayrıca sadece denklemlere dayalı Heyting cebiri tanımlamak da mümkündür.

Teorem 3.2.2 $L$ bir (dağılmalı) kafes olsun. $L$ nin bir Heyting cebiri olması için bir gerek ve yeter koşul $L$ üzerinde aşağıdakilerin gerçeklendiği bir $\rightarrow$ ikili işlemin olmasıdır: her $a, b, c \in L$ için

1. $a \rightarrow a=1$;

2. $a \wedge(a \rightarrow b)=a \wedge b$

3. $b \wedge(a \rightarrow b)=b$

4. $a \rightarrow(b \wedge c)=(a \rightarrow b) \wedge(a \rightarrow c)$.

Kanıt Koşul Gerektirir: $L$ bir Heyting cebiri ve $a, b, c \in L$ olsun.

1. $a \wedge c \leq a$ olduğu açıktır ve Tanım 3.2.1 gereği $c \leq a \rightarrow a$ olarak yazılır. Bu ise $a \rightarrow a=1$ e denktir.

2. $a \wedge b \leq b$ olduğu açıktır ve Tanım 3.2.1 uyarınca $b \leq a \rightarrow b$ ye denktir. Her kafeste monotonluk yasaları ( $a \leq b$ ve $c \leq d$ ise $a \wedge c \leq b \wedge d$ ve $a \vee c \leq b \vee$ d) geçerli olduğu için $b \leq a \rightarrow b, a \wedge b \leq a \wedge(a \rightarrow b)$ yi gerektirir. Diğer eşitsizlik için $a \rightarrow b=\bigvee\{c \in H: a \wedge c \leq b\}$ ile birlikte supremum (burada $\bigvee$ ile gösteriliyor) tanımı $a \rightarrow b \leq b$ yi verir. Bir kez daha monotonluktan $a \wedge(a \rightarrow$ 
b) $\leq a \wedge b$ elde edilir ve böylece ikinci eşitliği gösterilmiş olur.

3. $b \wedge(a \rightarrow b) \leq b$ olduğu açıktır. Ters yön için $b=\inf \{b, a \rightarrow b\}$ olduğunu göstermek gerekir. Şimdi $b \leq b$ açıktır ve Tanım 3.2.1 gereğince $b \leq a \rightarrow b$, $a \wedge b \leq b$ ye denktir. Böylece $b \wedge(a \rightarrow b)=b$ dir.

4. Benzer şekilde ispatlanır.

Koşul Yeterdir: L teoremin ifadesinde yer alan dört koşulu sağlayan bir kafes ve $a, b, c \in L$ olsun.

$c \leq a \rightarrow b$ varsayalım. O zaman monotonluktan $a \wedge c \leq a \wedge(a \rightarrow b)$ ve 2. den $a \wedge c \leq a \wedge b$ elde edilir. Ama $a \wedge b \leq b$ dir, böylece $a \wedge c \leq b$ elde edilir.

$a \wedge c \leq b$ varsayalım. O zaman $a \wedge c=(a \wedge a) \wedge c=a \wedge(a \wedge c) \leq a \wedge b=a \wedge$ $(a \rightarrow b)$, sirasıyla eşgüçlülük, hipotez ve 2. den yazılır. Her dağılmalı kafeste sadeleştirme yasası $(a \wedge b=a \wedge c$ ve $a \vee b=a \vee c$ ise $b=c$ dir) geçerli olduğu için $c \leq a \rightarrow b$ bulunur.

Burada Heyting cebirleri, Boole cebirleri ve kafesler arasında var olan ilişkileri açıklayan örnekler veriliyor.

Örnek 3.2.3 Her Boole cebiri bir Heyting cebiridir. Bunun için $a \rightarrow b:=a^{\prime} \vee b$ almak yeterlidir.

Örnek 3.2.4 Gerektirme işlemi

$a \rightarrow b:=\left\{\begin{array}{lc}b, & a>b \text { ise } \\ 1, & \text { aksi halde }\end{array}\right.$

gibi tanımlanırsa, her tam sıralı küme bir Heyting cebiridir.

Her Heyting cebiri bir Boole cebiri değildir. Gerçekten, $\rightarrow$ işlemi Örnek 3.2.4 deki gibi tanımlandığında, Boole cebiri olmayan en basit Heyting cebiri tam siralı

$\mathbf{H}=\{0,1 / 2,1\}$ kümesidir: $\mathbf{H}$ üzerinde $\mathrm{v}, \wedge, \rightarrow$, ve ' işlemleri aşağı çizelgelerdeki gibi tanımlandığında, Boole cebirlerinde geçerli olan üçüncünün olmazlığı yasası

$(1 / 2 \vee(1 / 2))^{\prime}=1 / 2 \vee(1 / 2 \rightarrow 0)=1 / 2 \vee 0=1 / 2$

nedeniyle yanlışlanır.

\begin{tabular}{|c|c|c|c|c|c|c|c|c|c|c|c|c|c|}
\hline$\wedge$ & 0 & $1 / 2$ & 1 & $\vee$ & 0 & $1 / 2$ & 1 & $\rightarrow$ & 0 & $1 / 2$ & 1 & & \\
\hline 0 & 0 & 0 & 0 & 0 & 0 & $1 / 2$ & 1 & 0 & 1 & 1 & 1 & 0 & 1 \\
\hline $1 / 2$ & 0 & $1 / 2$ & $1 / 2$ & $1 / 2$ & $1 / 2$ & $1 / 2$ & 1 & $1 / 2$ & 0 & 1 & 1 & $1 / 2$ & 0 \\
\hline 1 & 0 & $1 / 2$ & 1 & 1 & 1 & 1 & 1 & 1 & 0 & $1 / 2$ & 1 & 1 & 0 \\
\hline
\end{tabular}

Örnek 3.2.5 Her sonlu dağılmalı kafes bir Heyting cebiridir.

Bunu göstermek için $L$ sonlu, dağılmalı bir kafes olmak üzere 
$a \rightarrow b:=\bigvee\{c \in L: c \wedge a \leq b\}$

almak yeterlidir.

\section{Örnek 3.2.6}

- $\tau, \mathbb{R}^{2}$ nin tüm açık kümelerinin ailesi;

- $\quad U$ ve $\cap$ işlemleri küme birleşim ve küme kesişim;

- Her $U, V \in \tau$ için $I$ iç operatörü ve ' kümesel tümleyen olmak üzere

$$
U \rightarrow V:=I\left(U^{\mathrm{c}} \cup V\right) ;
$$

- $U^{\prime}=I\left(U^{\prime}\right)$

- $0=\emptyset$ ve $1=\mathbb{R}^{2}$

olmak üzere $\mathbb{H}=\langle\tau, \quad \cup, \cap, \quad \rightarrow, \quad, \quad 0,1\rangle$ bir Heyting cebiridir.

Bu örnekte gösterilmeye değer tek özellik $U, V, W \in \tau$ için

$U \cap W \subseteq V \Leftrightarrow W \subseteq U \rightarrow V$

denkliğidir. Önce her $U, V, W$ için

$U \cap W \subseteq V \Leftrightarrow W \subseteq U^{c} \cup V$

denkliğini gösterelim. $U \cap W \subseteq V$ olsun ve $W \nsubseteq U^{c} \cup V$ varsayalım. O zaman öyle bir $w \in W$ elemanı vardır ki $w \notin U^{c} \cup V$ olsun. Buradan $w \notin U^{c}$ ve $w \notin V$, dolayısiyla $w \in U$ dur. Böylece $w \in U \cap W \subseteq V$ den $w \in V$ sonuçlanır ki bu $w \notin V$ ile çelişir.

Şimdi $W \subseteq U^{c} \cup V \Leftrightarrow W \subseteq I\left(U^{c} \cup V\right)$ denkliğini kanıtlamak için bir gözlemde bulunalım. $X$ bir açık küme ise $X \subseteq Y, X \subseteq I(Y)$ yi gerektirir. Böylece, $X=W$ alırsak, $\Rightarrow$ yönü gösterilmiş olur. Ters yön için ise $I\left(U^{c} \cup V\right) \subseteq U^{c} \cup V$ olduğunu not etmek yeterlidir.

\section{Sezgisel önermeler lojiği için modeller}

Sezgisel önermeler lojiği için semantiklere geçmeden önce bir noktanın açılanması gerekmektedir. IPC nin karakteristik özelliklerinden biri her sezgisel önermenin doğru ya da yanlış olmamasıdır; bu özellik IPC yi CP den ayıran en temel karakteristiktir. Alt kümeler bağlamında bu, her alt kümenin bir tümleyene sahip olmayabileceği anlamına gelir. Örneğin, $P(\{a, b\})$ kuvvet kümesinin $H=\{\varnothing, \quad\{a\},\{a, b\}\}$ alt kümesi $0=\emptyset$ ve $1=\{a, b\}$ seçkin elemanlı ve $\cup, \cap$ işlemli dağılmalı bir kafestir. Ancak $\{a\}$ elemanının $\{a\} \cap A=0$ ve $\{a\} \cup A=1$ olacak şekilde bir $A$ tümleyeni yoktur.

\subsection{IPC için Kripke semantiği}

IPC için bir Kripke modeli bir $\mathcal{M}=\langle W, \quad R, \quad V\rangle$ üçlüsünden oluşur; burada $W$ olası dünyaların boştan farklı bir kümesi; $R, W$ üzerinde bir önsıralama (yani, yansıyan ve geçişken) bağıntısı ve $V, \mathbb{P}$ den $\mathcal{P}(W)$ kuvvet kümesine tanımlı değerlendirme 
fonksiyonudur. Bazen $V$ fonksiyonu $V: \mathbb{P} \times W \rightarrow\left\{\begin{array}{ll}0, & 1\end{array}\right\}$ şeklinde de tanımlanır. Burada $V$ fonksiyonunun $R$ bağıntısına göre monoton olması istenir: her $w, u \in W$ için $w R u$ ise $V(p, w) \leq V(p, u)$ dir. Diğer bir deyişle, $V(p, w)=1$ ve $w R u$ ise $V(p, u)=1$ dir. $V$ fonksiyonuna $\ddot{u} s$ kapalı (upper closed) fonksiyon da denir. $R(w):=\{u \in$ $W: w R u\}$ tanımını yapalım.

Bir formül farklı dünyalarda doğru ya da yanlış olabilir. $\varphi$ formülü modelin bir $w$ dünyasında doğru ise bu $\mathcal{M}, w \vDash \varphi$ ile gösterilir; $\vDash$ (ya da $\vDash$ ) bağıntısına zorlama bağıntısı (forcing relation) denir ve aşağıdaki gibi tanımlanır:

- $\mathcal{M}, w \vDash p$ ancak ve ancak $V(p, w)=1$ ya da $w \in V(p)$.

- $\mathcal{M}, w \vDash \neg \varphi$ ancak ve ancak her $u \in R(w)$ için $\mathcal{M}, w \not \models \varphi$.

- $\mathcal{M}, w \vDash \varphi \wedge \psi$ ancak ve ancak $\mathcal{M}, w \vDash \varphi$ ve $\mathcal{M}, w \vDash \psi$.

- $\mathcal{M}, w \vDash \varphi \vee \psi$ ancak ve ancak $\mathcal{M}, w \vDash \varphi$ ya da $\mathcal{M}, w \vDash \psi$.

- $\mathcal{M}, w \vDash \varphi \rightarrow \psi$ ancak ve ancak her $u \in R(w)$ için ya $\mathcal{M}, w \not \varphi$ ya da $\mathcal{M}, w \vDash \psi$. Ya da, denk olarak, ancak ve ancak her $u \in R(w)$ için $\mathcal{M}, w \vDash \varphi$, $\mathcal{M}, w \vDash \psi$ yi gerektirir.

$\mathrm{Bu}$ tanım zorlama bağıntısının monotonluğunu, yani $\mathcal{M}, w \vDash \varphi$ ve $u \in R(w)$ ise $\mathcal{M}, u \vDash \varphi$, korumak amaciyla tasarlanmıştır.

\section{Uyarılar 4.1.1}

1. Kripke modelinin evreni $W$ yalnız bir olası dünyadan oluşuyorsa o zaman model $\mathrm{CP}$ için bir modele dönüşür.

2. $V$ fonksiyonu üst kapalı idi: $\mathcal{M}, w \vDash \varphi$ ve $u \in R(w)$ ise $\mathcal{M}, u \vDash \varphi$ dir. Şimdi $w \in R(u)$ ve $u \in R(w)$ olsun. O zaman $\mathcal{M}, w \vDash \varphi$ ancak ve ancak $\mathcal{M}, u \vDash \varphi$ dir. Bu durum, formüllerin geçerliliği bağlamında $R$ bağıntısının bir kısmi sıralama bağıntısı olarak alınabileceğini gösterir.

Bir lojiğin bir semantiğe göre sağlam (sound) olduğunu göstermek için aksiyomlarının o semantik altında geçerli olduğu ve çıkarım kurallarının geçerliliği koruduğu gösterilmelidir. $\mathrm{Bu} \varphi$ formülü IPC ya da Int in bir teoremi ise bu $\vdash_{I n t} \varphi$ ile gösterilir.

Teorem 4.1.2 (Sağlamlı Teoremi) $\mathcal{M}=\langle W, \quad R, \quad V\rangle$ herhangi bir Kripke modeli ve $w, W$ nin herhangi bir dünyası olsun. Eğer $\vdash_{\text {Int }} \varphi$ ise o zaman $\mathcal{M}, w \vDash \varphi$ dir.

Kanıt Önce modus ponens (MP) çıkarım kuralının geçerliliği koruduğunu gösterelim. $\mathcal{M}, w \vDash \varphi$ ve $\mathcal{M}, w \vDash \varphi \rightarrow \psi$ verilsin. O zaman her $u \in R(w)$ için ya $\mathcal{M}, u \notin \varphi$ ya da $\mathcal{M}, u \vDash \psi$ dir. $R$ bağıntısı yansıyan olduğu için $u=w$ alabiliriz. Böylece $\mathcal{M}, u \vDash$ $\varphi$ verildiğinde $\mathcal{M}, w \vDash \psi$ sonuçlanmak zorundadır.

Aksiyomların bu semantiğe göre geçerli olduğunu gerçeklemek için en kapsamlı olanı, $(\varphi \rightarrow(\psi \rightarrow \chi)) \rightarrow((\varphi \rightarrow \psi) \rightarrow(\varphi \rightarrow \chi))$, ele alacağız; diğerleri benzer şekilde çalışılır. $\alpha \rightarrow \beta$ biçiminde bir formülün bir $w$ dünyasında doğru olduğu, her $u \in R(w)$ için $\mathcal{M}, u \vDash \alpha$ ise $\mathcal{M}, u \vDash \beta$ olduğu gösterilir. Buna göre her hangi bir $u \in R(w)$ için $\mathcal{M}, u \vDash \varphi \rightarrow(\psi \rightarrow \chi)$ varsayalım. Gösterilmesi gereken $\mathcal{M}, u \vDash(\varphi \rightarrow \psi) \rightarrow$ $(\varphi \rightarrow \chi)$ dir. Bir kez daha $\mathcal{M}, v \vDash \varphi \rightarrow \psi$ olacak şekilde keyfi bir $v \in R(u)$ dünyası verilsin ve $\mathcal{M}, v \vDash \varphi \rightarrow \chi$ olduğunu gösterelim. 
Nihayet $\mathcal{M}, w \vDash \varphi$ olacak şekilde $w \in R(v)$ dünyası verilsin ve $\mathcal{M}, w \vDash \chi$ olduğunu gösterelim. $R$ bağıntısının yansıyan, geçişken oluşundan ve $V$ fonksiyonunun monotonluğundan, $u \in R(w)$ ve $v \in R(w)$ nedeni ile $\mathcal{M}, w \vDash \varphi \rightarrow(\psi \rightarrow \chi)$ ve $\mathcal{M}, w \vDash \varphi \rightarrow \psi$ dir. $\quad$ MP kuralı $\vDash$ için uygulanabilir olduğundan $\mathcal{M}, w \vDash \psi$ ve $\mathcal{M}, w \vDash \psi \rightarrow \chi \operatorname{den} \mathcal{M}, w \vDash \chi$ sonuçlandırilır.

Bu teorem bir formülün Int de türetilemediğinin bir kanıtı olarak kullanılır.

Örnek 4.1.3 $\vdash_{C P} p \vee \neg p$ olduğu iyi bilinmektedir. Ancak $\nvdash_{\text {Int }} p \vee \neg p$, dolayısıyla $p \vee \neg p$ önermesinin Int de geçerli olmadığını kanıtlamak için Kripke modelinin en az iki elemanlı bir evrene sahip olması gerekir. $W=\{w, u\}$ ve $R, W$ üzerinde yansıyan olsun. $V(p)=\{u\}$, yani $V(p, u)=1$ ve $V(p, w)=0$ alırsak, $\mathcal{M}, w \not p$ ve $\mathcal{M}, w \not \neq$ $\neg p$ dir çünkü $u \in R(w)$ için $\mathcal{M}, u \vDash p$ dir. O halde $\mathcal{M}, w \notin p \vee \neg p$ elde edilir. Teorem 4.1.2 nin karşıt tersinden $\nvdash_{\text {Int }} p \vee \neg p$ sonuçlanır.

Sağlamlık teoreminin tersi olan tamlık teoremi (completeness theorem) her hangi bir Kripke modelinin her dünyasında doğru olan bir formülün Int de kanıtlanabilir olduğunu ifade eder.

Teorem 4.1.4 (Tamlık Teoremi) Her hangi bir Kripke modeli $\mathcal{M}=\langle W, \quad R, \quad V\rangle$ ve her $w \in W$ için $\mathcal{M}, u \vDash \varphi$ ise $\vdash_{\text {Int }} \varphi$ dir.

Kanıt için [5] kaynağına bakılabilir.

\subsection{IPC için Heyting cebiri modeli}

Teorem 4.2.1 (Sağlamlık Teoremi) $\mathbb{H}$ bir Heyting cebiri, $\Gamma$ formüllerin sonlu bir kümesi ve $V: \mathbb{P} \rightarrow \mathbb{H}$ bir değerlendirme fonksiyonu olsun. $\Gamma \vdash_{I n t} \varphi$ ise o zaman $\Gamma \vdash_{\mathbb{H}} \varphi$ dir.

Kanıt IPC nin kurallarının Heyting gerektirmesini koruduklarını göstermemiz gerekmektedir.

$\Gamma \vdash_{\text {Int }} \varphi$ türetiminde uygulanan son kuralın $(\wedge I)$ olduğunu varsayalım. $\mathrm{O}$ zaman $\varphi=\psi \wedge \chi$ dir ve $\Gamma_{1} \subseteq \Gamma, \Gamma_{2} \subseteq \Gamma$ alt kümeleri için $\Gamma_{1} \vdash_{\text {Int }} \psi$ ve $\Gamma_{2} \vdash_{\text {Int }} \chi$ türetimleri vardır; yani $\mathrm{V}\left(\wedge \Gamma_{1}\right) \leq V(\psi)$ ve $\mathrm{V}\left(\wedge \Gamma_{2}\right) \leq V(\chi)$ dir. Buradan $V(\wedge \Gamma) \leq \mathrm{V}\left(\wedge \Gamma_{1}\right) \wedge$ $\mathrm{V}\left(\wedge \Gamma_{2}\right) \leq V(\psi) \wedge V(\chi) \leq V(\psi \wedge \chi)$ elde edilir ve böylece $(\wedge I)$ kuralı korunmuş olur.

$\Gamma \vdash_{\text {Int }} \varphi$ türetiminde son uygulanan kuralın $(\rightarrow I)$ olduğunu varsayalım. O zaman $\varphi=\psi \rightarrow \chi$ ve $\Gamma \cup\{\psi\} \vdash_{\text {Int }} \chi$ dir; böylece tümevarım hipotezinden $V(\wedge \Gamma) \wedge V(\psi)=$ $V(\wedge \Gamma \cup\{\psi\}) \leq V(\chi)$ olur. Ama Heyting cebirinde $\rightarrow$ işleminin tanımından ve değerlendirme fonksiyonun tanımından $V(\wedge \Gamma) \leq V(\psi) \rightarrow V(\chi)=V(\psi \rightarrow \chi)$ elde edilir, dolayısıyla $(\rightarrow I)$ kuralı korunmuş olur. Kalan kurallar Heyting cebirinin karşılık gelen özellikleri kullanılarak benzer şekilde korunur.

Teorem 4.2.2 (Tamlık Teoremi) $\varphi$ formülü IPC de kanıtlanabilirdir ancak ve ancak her bir Heyting cebiri $\mathbb{H}$ için $\varphi \mathbb{H}$-geçerlidir; yani

$\vdash_{\text {Int }} \varphi \Leftrightarrow \vdash_{\mathbb{H}} \varphi$

dir. 
Kanıt Koşul Gerektir: $\varphi$, IPC de kanıtlanabilir ise $\vdash_{\text {Int }} \varphi$ nin bir türetimi vardır. O halde Teorem 4.2.1 uyarınca herhangi bir $\mathbb{H}$-değerlendirme $V$ için $1 \leq V(\varphi)$ dir ve böylece $\varphi, \mathbb{H}$-geçerlidir.

Koşul Yeterdir: $\mathbb{F}_{i}$, IPC formüllerinin kümesi olsun ve $\mathbb{F}_{i}$ üzerinde $(\varphi \sim \psi) \Leftrightarrow$ $\vdash_{\text {Int }}(\varphi \leftrightarrow \psi)$ denklik bağıntısını tanımlayalım. $\mathbb{H}=\mathbb{F}_{i} / \sim$, bağıntısına göre

$[\varphi]=\left\{\psi \in \mathbb{F}_{i}: \varphi \sim \psi\right\}$ denklik sinıflarının kümesi olsun. $\mathbb{H}$ üzerinde $[\varphi] \sim[\psi] \Leftrightarrow$ $\vdash_{\text {Int }} \varphi \rightarrow \psi$ kısmi siralama bağıntısını tanımlayalım. $\mathbb{H}$ üzerinde $[\varphi] \wedge_{\mathbb{H}}[\psi]=[\varphi \wedge$ $\psi],[\varphi] \vee_{\mathbb{H}}[\psi]=[\varphi \vee \psi]$ ve $[\varphi] \rightarrow_{\mathbb{H}}[\psi]=[\varphi \rightarrow \psi]$ şeklinde tanımlanan işlemlerin temsilci elemandan bağımsız olduğu ve $0_{\mathbb{H}}=[0], 1_{\mathbb{H}}=[1]$ alındığında $\left\langle\mathbb{H}, \quad \leq, \quad \vee_{\mathbb{H}}, \quad \Lambda_{\mathbb{H}}, \quad \rightarrow_{\mathbb{H}}, 0_{\mathbb{H}}, 1_{\mathbb{H}}\right\rangle$ yapısının bir Heyting cebiri oluşturduğu kolayca gösterilebilir. Şimdi $V(\varphi)=1_{\mathbb{H}}$ ise o zaman $\vdash_{I n t} \varphi \leftrightarrow 1$ dir ve böylece $\vdash_{\text {Int }} \varphi$ elde edilir.

$\mathrm{Bu}$ semantiğe göre $\mathrm{CP}$ de geçerli ancak IPC de geçerli olmayan formül örnekleri verilebilir.

Örnek 4.2.3 $H=\{\varnothing, \quad\{a\}, \quad\{a, b\}\} \subseteq \mathcal{P}(\{a, b\})$ kümesi $\cup$ ve $\cap$ işlemleri altında $0=\emptyset$ ve $1=\{a, b\}$ seçkin elemanlı dağılmalı bir kafestir. $p \in P$ için $V(p)=\{a\}$ olsun. O zaman $V(\neg p)=\neg\{a\}=\emptyset$ dir.

$\forall_{\text {Int }} \neg \neg p \rightarrow p$ dir, çünkü dir.

$$
V(\neg \neg p \rightarrow p)=V(\neg \neg p) \rightarrow V(p)=1 \rightarrow V(p)=V(p)=\{a\} \neq\{a, \quad b\}=1
$$

$\vdash_{\text {Int }} \neg \neg p \rightarrow p$ dir, çünkü

dir.

$$
V(\neg p \vee \neg \neg p)=V(\neg p) \vee V(\neg \neg p)=\emptyset \cup\{a, \quad b\}=1
$$

\subsection{IPC için topolojik modeller}

Her formülün değerlendirmesi bir açık küme olacak şekilde, IPC nin formüllerini bir $\langle X, \quad \tau\rangle$ topolojik uzayının alt kümeleri olarak yorumlayacağız. Önerme değişkenleri için $V: \mathbb{P} \rightarrow \tau$ fonksiyonunun formüllere genişletilmesi, yine aynı $V$ kullanılarak, $V: \mathbb{F}_{i} \rightarrow \tau$ olarak tanımlanacaktır. $\quad \varphi, \psi \in \mathbb{F}_{i}$ için $V(\varphi \wedge \psi)=V(\varphi) \cap V(\psi)$ ve $V(\varphi \vee \psi)=V(\varphi) \cup V(\psi)$ kümeleri $\tau$ kümesine aittir. Ancak $V(\varphi \rightarrow \psi)$ kümesi $(X-$ $V(\varphi)) \cup V(\psi)$ gibi tanımlanırsa, $\rightarrow$ işlemi hatalı tanımlanmış olur çünkü $(X-V(\varphi)) \cup$ $V(\psi)$ bir açık küme olmayabilir. Uygun tanım $V(\varphi \rightarrow \psi)=I(X-V(\varphi)) \cup V(\psi)$ dir. Nihayet $\quad V(\perp)=\emptyset \quad$ olmak üzere, $\quad V(\neg \varphi)=V(\varphi \rightarrow \perp)=I(X-V(\varphi)) \cup V(\perp)=$ $I(X-V(\varphi))$ olarak tanımlanacaktır.

Bir $\langle X, \tau\rangle$ topolojik uzayı üzerindeki $V$ değerlendirmesi altında $V(\varphi)=X$ ise $\varphi$ formülü doğru olarak değerlendirilir. Bu yorum altında üçüncünün olmazlığı yasası geçerli değildir. Gerçekten, $\mathbb{R}^{n}$ deki bir $\varphi$ açık kümesi (örneğin bir açık yuvar) ne $\varphi$ ye ne de tümleyeninin içine, $I\left(\mathbb{R}^{n}-\varphi\right)$, ait noktalardan oluşan bir sınıra sahiptir; bir sınır noktasının her komşuluğu $\varphi$ nin ve tümleyeninin noktalarını içerir.

Bir topolojik model bir $\mathcal{M}=\langle W, \quad R, \quad V\rangle$ üçlüsüdür. 
Teorem 4.3.1 (Sağlamlık Teoremi) $\vdash_{\text {Int }} \varphi$ ise o zaman $\varphi$, her $\mathcal{M}=\langle W, \quad R, \quad V\rangle$ topolojik modelinde doğrudur.

Kanıt Önce MP kuralının geçerliliği koruduğunu gösterelim: $V(\varphi)=X$ ve $V(\varphi \rightarrow$ $\psi)=X$ ise $V(\psi)=X$ dir. Ama bu açıtır: $V(\varphi \rightarrow \psi)=I(X-V(\varphi)) \cup V(\psi)=$ $I(X-X) \cup V(\psi)=I(\varnothing) \cup X=\emptyset \cup X=X$.

IPC nin aksiyomlarından sadece $\varphi \rightarrow(\psi \rightarrow \varphi)$ aksiyomunu değerlendirelim; kalan aksiyomlar benzer şekilde çalışılır. Göstermemiz gereken $V(\varphi \rightarrow(\psi \rightarrow \varphi))=X$ olduğudur. Öncelikle $V(\varphi \rightarrow(\psi \rightarrow \varphi)) \subseteq X$ açıtır. Diğer kapsama için $\rightarrow$ gerektirmesinin yorumunu ve $I$ iç operatörünün monotonluğunu (yani $A \subseteq B$ ise $I(A) \subseteq I(B))$ kullanmak yeterlidir.

$$
\begin{aligned}
V(\varphi \rightarrow(\psi \rightarrow \varphi)) & =I(X-V(\varphi)) \cup V(\psi \rightarrow \varphi) \\
& =I(X-V(\varphi)) \cup I(X-V(\psi)) \cup V(\varphi) \\
& \supseteq I(X-V(\varphi)) \cup V(\varphi) \\
& \supseteq X
\end{aligned}
$$

olup, $V(\varphi \rightarrow(\psi \rightarrow \varphi))=X$ dir ve $\varphi \rightarrow(\psi \rightarrow \varphi)$ aksiyomu bu semantik altında geçerlidir.

(Okur $\quad(\varphi \rightarrow(\psi \rightarrow \chi)) \rightarrow((\varphi \rightarrow \psi) \rightarrow(\varphi \rightarrow \chi)) \quad$ aksiyomunun geçerliliğini denetlemek isteyebilir!)

IPC nin topolojik semantiğe göre tamlığını ilk kez ifade eden ve kanıtlayan McKinsey ve Tarski [6] dir.

Teorem 4.3.2 (Tamlık Teoremi) Her $n \geq 1$ için $\vdash_{\text {Int }} \varphi$ ancak ve ancak standart topoloji $\mathbb{R}^{n}$ üzerindeki her $V$ değerlendirmesi için $V(\varphi)=\mathbb{R}^{n}$ dir.

Sonuç 4.3.3 Bu çalışmada sezgisel önermeler lojiği için bilinen Kripke, Heyting ve Topolojik semantikler ayrıntılı ele alınmış, anlaş1lır örneklerle, bu semantiklere göre, IPC yi CP den ayırt eden özelliklere vurgu yapılmıştır. IPC için son zamanlarda çalışılan bir diğer semantik komşuluk (neighborhood) semantiğidir. Bu konuda $[4,7,11]$ kaynaklarına bakılabilir.

\section{Kaynaklar}

[1] Bezhanishvili, N. ve de Jong, D., Intuitionistic Logic, ILLC, Universiteit van Amsterdam, (2010).

[2] Brown, C. E., Semantics of intuitionistic propositional logic, Heyting algebras and Kripke models, (2014).

[3] Kato, T., Boolean algebra and Propositional Logic, June 23, (2015).

[4] Kojima, K., Relational and neighborhood semantics for intuitionistic modal logic, Reports on Mathematical Modal Logic, 47, 87-113, (2012).

[5] Kuznetsov, S., Lecture Notes on Logic, University of Pennsylvania, (2017).

[6] McKinsey, J.C.C. ve Tarski, A., The algebra of topology, Annals of 
Mathematics, 141-191, (1944).

[7] Moniri, M. ve Maleki, F.S, Neighborhood semantics for basic and intuitionistic logic, Logic and Logical Philosophy, 24, 334-355, (2015).

[8] Palmgren, E., Semantics of Intuitionistic Propositional Logic, Lecture Notes for Applied Logic, Dept. of Math. Uppsala University, (2009).

[9] Troelstra, A.S. ve van Dalen, D., Constructivism in Mathematics: An Introduction, Volume 2, North-Holland, (1988).

[10] Van Dalen, D., Logic and Structure, Springer, (2012).

[11] Witczak, T., Intuitionistic modal logic based on neighborhood semantics without superset axiom, arXiv:1707.03859v2[math L0], (2017). 